\title{
La formation d'eau dense en Méditerranée : interactions d'échelles, changement climatique et écosystèmes
}

\section{Résumé}

La Méditerranée nord-occidentale (MNO) constitue un formidable laboratoire d'étude des deux processus responsables de la formation d'eau dense dans l'océan : le cascading et la convection profonde. Un outil de modélisation de la dynamique océanique en MNO et de son influence sur les écosystèmes marins a été mis en place. Nous avons quantifié la contribution des processus atmosphériques et océaniques de petite échelle dans la formation et le devenir de l'eau dense issue de la convection profonde. Le changement climatique pourrait induire un affaiblissement de la circulation thermohaline méditerranéenne, qui provoquerait une disparition du cascading et une légère intensification de la boucle microbienne.

\section{Abstract}

Dense water formation in the Mediterranean Sea: Scale interactions, climate change and ecosystems

The Northwestern Mediterranean Sea (NWMS) is an ideal case study of both processes responsible for dense water formation: cascading and oceanic deep convection. We developed a coupled modelling tool of the NWMS ocean dynamics and of its influence on marine ecosystems. It enabled us to quantify the contribution of small scale oceanic and atmospheric processes in the formation and spreading of dense water formed during deep convection. Climate change could induce a weakening of the Mediterranean thermohaline circulation. As a result, cascading would practically disappear and the microbial loop would slightly increase.

\section{Marine Herrmann}

CNRM-Game, Météo-France/CNRS

42, avenue Gaspard-Coriolis - 31057 Toulouse Cedex marine.herrmann@m4x.org

a circulation océanique thermohaline globale, surnommée « le tapis roulant océanique », correspond au transfert en surface d'eaux chaudes et légères de l'équateur vers les régions polaires, et au fond d'eaux froides et denses dans le sens inverse. Responsable d'échanges considérables de chaleur entre les pôles et l'équateur, la circulation thermohal ine joue un rôle majeur dans le fonctionnement du climat. La formation d'eaux denses dans certaines régions du globe et leur transfert vers I'océan profond en sont I' un des moteurs.

\section{À l'origine de la formation d'eau dense : convection profonde et cascading}

Ces masses d'eau dense sont formées à la surface suite à un forçage thermohalin intense (évaporation, refroidissement, formation de glace de mer). A u large, dans les régions de grande profondeur, ce processus correspond à la convection océanique profonde ( $\mathrm{M}$ arshall et Schott, 1999) : la densification de l'eau de surface déstabilise la colonne d'eau, provoque son mélange vertical, et aboutit à la formation d'une masse d'eau homogène et dense qui coule et se propage ensuite en profondeur. La convection profonde a lieu en mer du Labrador, dans les mers nordiques de Norvège et du Groenland, en A ntarctique (mers de Weddell et de Ross), et au large du golfe du Lion en M éditerranée. Des masses d'eau dense sont aussi formées sur certains plateaux continentaux de faible profondeur (Ivanov et al., 2004). Elles s'écoulent vers les profondeurs de l'océan global en cascadant le long du talus continental : c'est le phénomène de cascading. Des épisodes de cascading ont, par exemple, été observés le long du talus du golfe du Lion (Canals et al., 2006), où la voie principale d'écoulement de l'eau dense formée sur le plateau semble être le canyon de Cap Creus, situé à l'extrémité sud-ouest du plateau (figure 1).

Les eaux denses formées lors de la convection profonde et du cascading participent au fonctionnement du climat à plusieurs niveaux. D'une part, de façon directe, puisqu'elles forment les masses d'eau profondes de la circulation thermohaline. Par exemple, cascading et convection profonde en Méditerranée nord-occidentale (MNO) sont respectivement à l'origine de la formation de deux des principales masses d'eau de la circulation thermohaline méditerranéenne : I'eau méditerranéenne occidentale intermédiaire (WIW, Western Mediterranean Intermediate Water en anglais) et I' eau méditerranéenne occidentale profonde (WMDW, Western M editerranean Deep Water). 


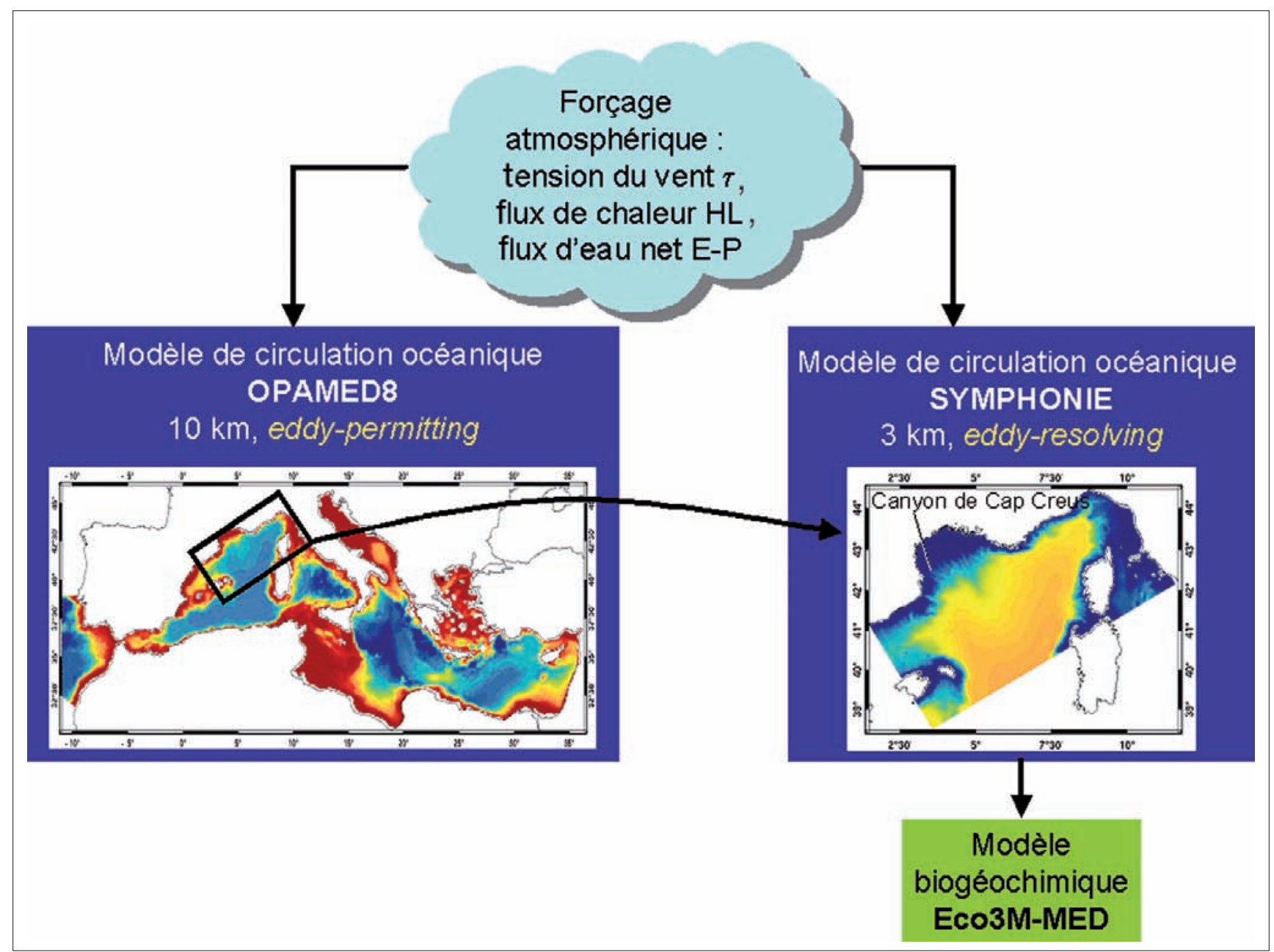

Figure 1 - Schéma de principe de l'outil de modélisation. Les domaines des modèles océaniques Opamed8 et Symphonie sont représentés. Les flèches indiquent les forçages atmosphère à océan ; océan à océan ; dynamique océanique à biogéochimie.

D'autre part, les eaux denses océaniques jouent un rôle important dans le cycle du carbone. Elles emportent en effet avec elles le carbone présent en surface sous forme particulaire ou dissoute vers les profondeurs de l'océan : c'est la pompe physique. Ces transferts verticaux entrent également en jeu dans le fonctionnement des écosystèmes marins : Iors du brassage vertical de la colonne d'eau associé à la convection profonde, des nutriments sont remontés du fond vers la surface, où a lieu la photosynthèse, c'est-à-dire la fixation du gaz carbonique dissous par le phytoplancton, premier maillon de la chaîne trophique : c'est la pompe biologique. A insi, ces apports en nutriments associés à la formation d'eau dense font de la M NO la région la plus productive de M éditerranée, mer globalement oligotrophe. Le cascading permet, quant à lui, d'apporter de la matière organique aux écosystèmes profonds.

La MNO constitue un formidable laboratoire pour l'étude de la convection profonde et du cascading : on peut mener dans cette région relativement facile d'accès des campagnes dédiées à l'observation de ces processus, ce qui est nettement plus ardu dans les régions polaires. On dispose ainsi d'une quantité de données relativement importantes sur la convection (citons entre autres Medoc-Group, 1970 ; Schott et L eaman, 1991 ; Leaman et Schott, 1991) et le cascading (Béthoux et al., 2002 ; Canals et al., 2006) en MNO. Ces campagnes ont permis de grandes avancées dans la connaissance de ces processus : zones et périodes de prédilection, importance des tourbillons de méso et submésoéchelle dans le transport des masses d'eau, variabilité et tendances des quantités et des caractéristiques hydrologiques de l' eau dense formée. Parallèlement aux observations, la modélisation numérique est un outil particulièrement adapté à l'étude de ces processus : elle permet d'étudier de façon continue toute la gamme des échelles spatio-temporelles en jeu, ainsi que leur interactions, en menant des tests de sensibilité à la prise en compte et la représentation de différents processus physiques. Les études numériques ont ainsi permis de mettre en évidence
I'importance du forçage atmosphérique et de sa fréquence (A rtale et al., 2002) ainsi que celle des tourbillons et méandres de mésoéchelle (M adec et al., 1991) dans le déroul ement de la convection profonde. De plus, la modélisation permet d'explorer des périodes passées ou futures inaccessibles aux observations.

Un grand nombre de questions se posent encore aujourd' hui sur la formation d'eau dense:

- Les études expérimentales et numériques existantes ont mis en évidence le rôle des interactions entre processus océaniques et atmosphériques de différentes échelles lors de la convection profonde. Est-il possible de quantifier le rôle des processus de petite échelle spatio-temporelle dans la formation et le devenir de la W MDW ?

- Les observations (M ertens et Schott, 1998 ; Heussner et al., 2006) ont montré que la formation d'eau dense en M NO, qu' elle ait lieu au large ou sur le plateau, présente une forte variabilité interannuelle, avec des années sans aucune formation d'eau dense, et des années où la couche de mélange océanique au large 
atteint le fond ( $\simeq 2000$ mètres en M NO). Quels sont les facteurs qui pilotent la variabilité de la formation et de l'exportation d' eau dense sur le plateau ?

- On observe depuis plusieurs décennies une augmentation constante de la température et de la salinité de l'eau profonde méditerranéenne (B éthoux et al., 2002). La formation d'eau dense en surface est liée en grande partie aux conditions atmosphériques, I'évolution des quantités d'eau dense formées et exportées vers le fond face aux variations climatiques à long terme est donc une question majeure. Somot et al. (2006) ont montré qu' au large, le changement climatique provoquerait une diminution du volume de WM DW formé lors de la convection profonde, et un affaiblissement significatif de la circulation thermohaline méditerranéenne. Qu'en sera-t-il du cascading ?

- Le fonctionnement des écosystèmes marins est fortement influencé par la dynamique océanique. Quel est le fonctionnement de l'écosystème pélagique planctonique, qui constitue le premier niveau de la chaîne trophique en $\mathrm{M} \mathrm{N} 0$, et quel rôle joue-t-il dans le cycle du carbone? La M NO est-elle un puits ou une source de carbone pour l'atmosphère ? Comment les caractéristiques de cet écosystème évolueront face aux variations climatiques ?

Pour répondre à ces questions, nous avons mis en place une stratégie de modélisation numérique de la dynamique océanique et de son influence sur l'écosystème planctonique pélagique en M NO. Un schéma de principe en est présenté figure 1. Le présent article propose une synthèse des résultats obtenus. Pour plus de détails, le lecteur est invité à se référer à $H$ errmann (2007), Herrmann et Somot (2008) et Herrmann et al. (2008a, 2008b).

\section{La convection}

\section{au large : interactions d'échelles et modélisation}

Pour étudier le rôle des interactions d'échelles et l'influence de la configuration des modèles sur la représentation de la convection, nous nous sommes appuyés sur l'étude d'un cas réel : I'hiver 1986-1987 en M NO. Cet hiver est particulièrement intéressant pour deux raisons. D'une part, il a été très froid et venteux, ce qui a donné lieu à un épisode de convection intense : la couche de mélange a atteint le fond, et la W M DW formée fait partie des plus denses des quarante dernières années du $x x^{e}$ siècle (M ertens et Schott, 1998). De plus, cet épisode a fait l'objet d'une campagne océanographique spécialement dédiée (Schott et Leaman, 1991 ; Leaman et Schott, 1991). On dispose donc de données qui présentent une couverture spatio-temporelle permettant de bien définir cet épisode en termes d'extension spatiale, de déroulement chronologique, de caractéristiques de la W M DW formée. Nous avons donc réalisé plusieurs simulations numériques de la période septembre 1986-septembre 1987 afin d'étudier et de quantifier le rôle des interactions d'échelles dans la convection.

\section{Les structures de mésoéchelle}

Pour examiner le rôle des structures de mésoéchelle, nous avons comparé deux simulations de résolutions spatiales différentes. La première, nommée EP87, a été effectuée sur I'ensemble du bassin avec le modèle océanique OPA à $1 / 8^{\circ}$ de résolution $(\simeq 10 \mathrm{~km})$ : ce modèle, dit eddy-permitting, ne permet pas de résoudre la mésoéchelle, de l'ordre de $10 \mathrm{~km}$ en MNO. La seconde, nommée ER87-1, a été effectuée sur un domaine couvrant la région de convection et de cascading (figure 1) au moyen du modèle Symphonie à $3 \mathrm{~km}$ de résolution : il permet de résoudre la mésoéchelle (eddyresolving). L es conditions initiales et aux limites latéral es ouvertes de ER87-1 sont données par les résultats de EP87. Pour les deux simulations, le forçage atmosphérique est issu de la réanalyse ERA 40 (Kalnay et al., 1996).

$L$ a différence principale entre EP87 et ER87-1 est liée à la différence de représentation des structures de mésoéchelle : de façon logique, elles ne sont pas reproduites dans EP87 alors qu'elles sont reproduites en accord avec les observations de Testor et Gascard (2006) dans ER87-1. La comparaison entre ces deux simulations permet donc d'en quantifier l'influence. L'analyse des flux de flottabilité et du spectre d'énergie cinétique, détaillées dans Herrmann et al. (2008a), montrent que ces structures facilitent les échanges latéraux d'eau et de flottabilité à la périphérie de la zone de convection, comme suggéré par $M$ arshall et Schott (1999) : de l'eau légère et stratifiée, associée à une flottabilité positive, est transportée depuis I' extérieur vers I'intérieur de la zone, et de l'eau dense, de flottabilité négative, est transportée dans le sens inverse. A u final, la comparaison de EP87 et ER 87-1 montre que ces structures jouent un rôle essentiel dans la formation et le devenir de la WM DW : d'une part, en important de l'eau légère dans la zone, elles limitent le volume $d^{\prime}$ eau dense finale $\left(\simeq 1,5 \times 10^{14} \mathrm{~m}^{3}\right)$ de $30 \%$; d'autre part, $78 \%$ de l'export total d'eau dense hors de la zone $\left(0,9 \times 1014 \mathrm{~m}^{3}\right)$ est dû aux structures de mésoéchelle. L a simulation ER 87-1 suggère que $66 \%$ de la WM DW exportée est entraînée dans le courant nord vers la mer Catalane pendant la convection, sans approfondissement. Les $34 \%$ restants sont exportés après la convection en profondeur (sous 1000 mètres) par des tourbillons vers le sud-est du bassin occidental. Ces valeurs sont comparables à celles obtenues grâce aux observations : Testor et Gascard (2006) estiment à $40 \%$ la contribution des tourbillons de mésoéchelle vers le sud-est, et Send et al. (1996) suggèrent que $50 \%$ de l'export pourrait être attribué à I'entraînement dans le courant nord.

\section{Les événements atmosphériques intenses}

Dans les deux simulations, la zone de convection est trop grande par rapport aux observations (figure 2). Ce défaut pourrait être lié à la faible résolution ( $~ 125 \mathrm{~km}$ ) du forçage atmosphérique issu d'ERA 40 : celle-ci serait à l' origine de la sous-estimation de la perte de chaleur hivernale observée dans ERA 40, corrigée dans EP87 et ER87-1 en ajoutant un terme correctif de -130 W.m-2 entre décembre et mars dans tout le bassin. Pour vérifier cette hypothèse, une simulation jumelle, ER87-2, a été effectuée en utilisant un forçage atmosphérique de plus haute résolution $(50 \mathrm{~km})$, obtenu en réalisant un downscaling dynamique d'ERA 40 avec le modèle atmosphérique A rpège-Climat. Cette méthode permet de corriger la sous-estimation de la perte de flottabilité observée dans ERA 40, tout en respectant la chronologie (figure 3). Cette correction n'est constante ni dans le temps ni dans l'espace, mais augmente pour les valeurs élevées : le downscaling améliore la représentation des extrêmes météorologiques spatiaux et temporels (figure 2 et Herrmann et Somot, 2008). Enf in, on a réal isé une troisième simulation, ER 87-3, dans laquelle on utilise les flux d'ERA 40 sans correction.

Si ERA 40 « corrigé » conduit à une surestimation de la taille de la zone de convection, ERA40 sans correction ne 


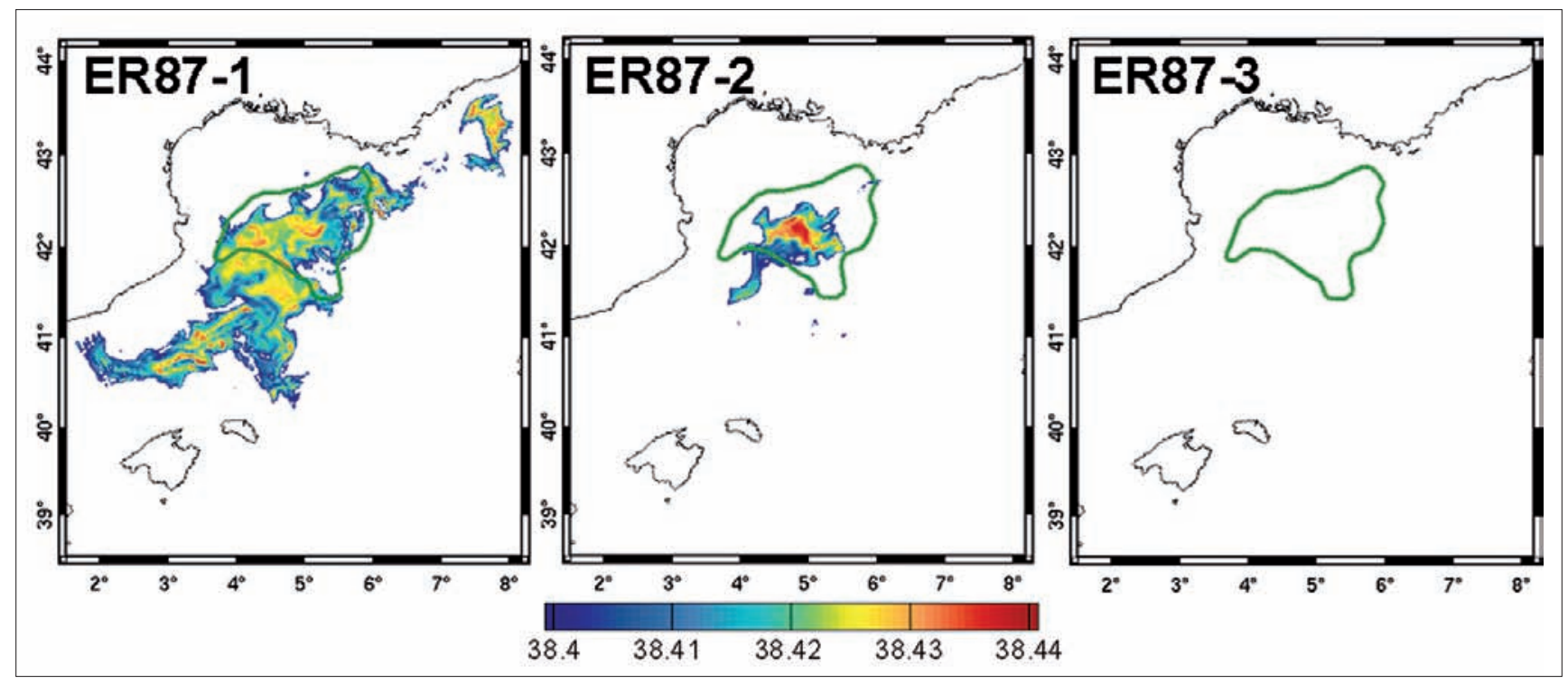

Figure 2 - Zone de convection modélisée dans ER87-1, ER87-2 et ER87-3 et observée le 20 février 1987, définie comme la zone où la salinité de surface dépasse 38,4. Les couleurs correspondent à la région modélisée ; le tracé vert montre la zone de convection observée par Leaman et Schott (1991).

produit pas de convection (figure 2). ER $87-2$ reproduit la convection de façon nettement plus réaliste : la taille et la position de zone de convection correspondent à la zone observée par Leaman et Schott (1991), figure 2. Ces résultats, détaillés dans Herrmann et Somot (2008), montrent que les événements atmosphériques intenses temporels ainsi que spatiaux jouent un rôle essentiel dans le déroulement et les caractéristiques spatiales de la convection. Prendre en compte ce rôle dans les simulations océaniques nécessite de disposer de flux atmosphériques de résolution suffisante. Le downscaling dynamique est bien adapté à la production de telles données.

\section{La formation d'eau dense sur le plateau : variabilité interannuelle et changement
climatique}

L'outil de modélisation mis en place pour l'étude de la convection profonde au large a ensuite été utilisé pour étudier la réponse du cascading à la variabilité interannuelle et au changement climatique. Une simulation nommée EPA 2 a été effectuée avec le modèle 0 pamed8 (version régionale sur la M éditerranée du modèle OPA ) sur I'ensemble du bassin sur la période 1960-2100 (Somot et al., 2006). EPA 2 a été forcée aux frontières

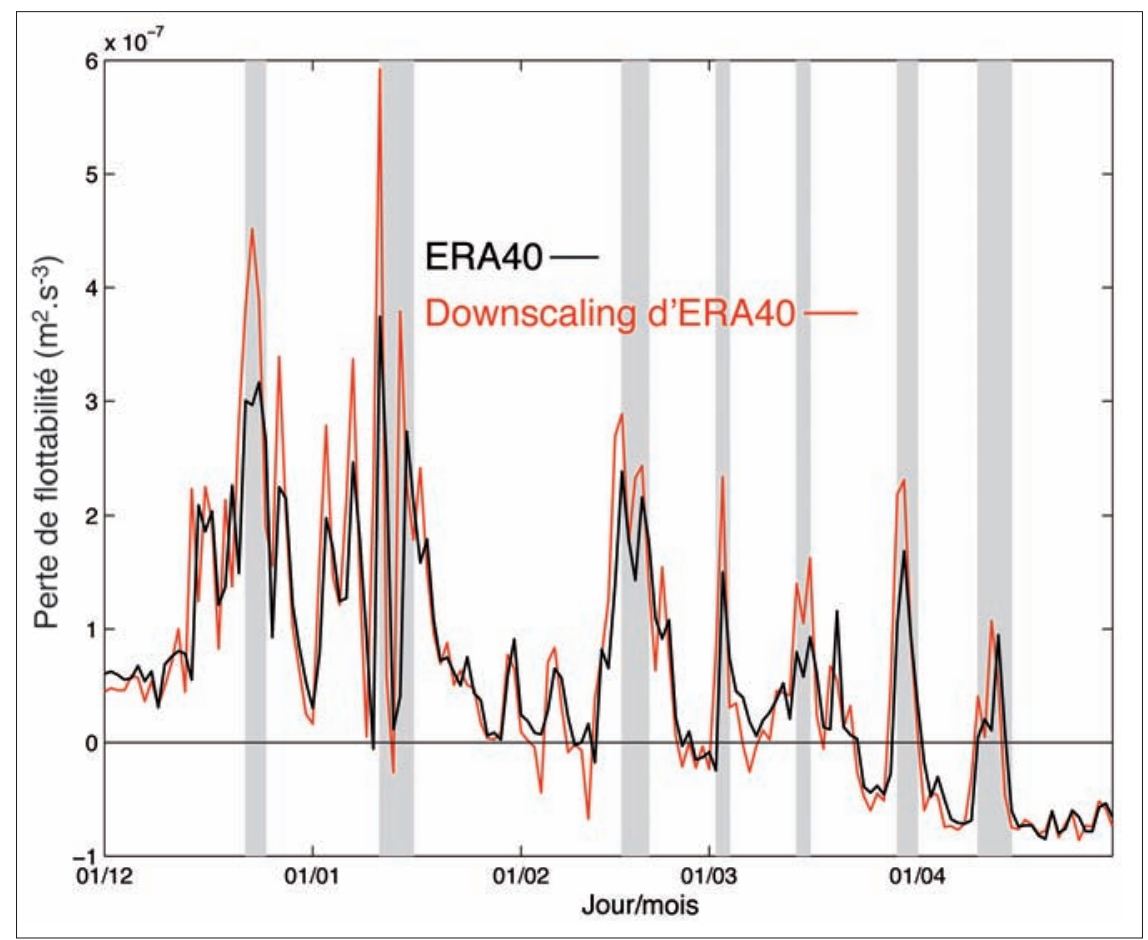

Figure 3 - Évolution quotidienne de la perte de flottabilité atmosphérique sur la zone d'étude pendant I'hiver 19861987 dans ERA40 (noir) et le downscaling d'ERA40 (rouge).

ouvertes (latérales et de surface) par les résultats d'une simulation couplée globale effectuée avec le modèle A rpègeClimat - OPA dans le cadre du Groupe intergouvernemental d'experts sur l'évolution du climat (Giec). Dans cette simulation couplée, la concentration des gaz à effet de serre est donnée par les observations jusqu' en 2000, puis suit les hypothèses du scénario $A 2$ du Giec. L'influence du changement climatique sur la convection profonde au large a été examinée dans EPA 2 par Somot et al. (2006). Ceux-ci concluent qu' en raison de la diminution de la perte de flottabilité moyenne annuelle, on pourrait assister en M éditerranée à une intensification de la stratification de la colonne d'eau qui entraînerait une diminution de la convection profonde et un affaiblissement de la circulation thermohaline. Pour examiner les effets sur le cascading, nous avons effectué des simulations eddy-resolving en utilisant la même méthode que pour ER 87 : le modèle Symphonie a été forcé aux frontières latérales par les résultats de EPA 2, et les forçages atmosphérique et 


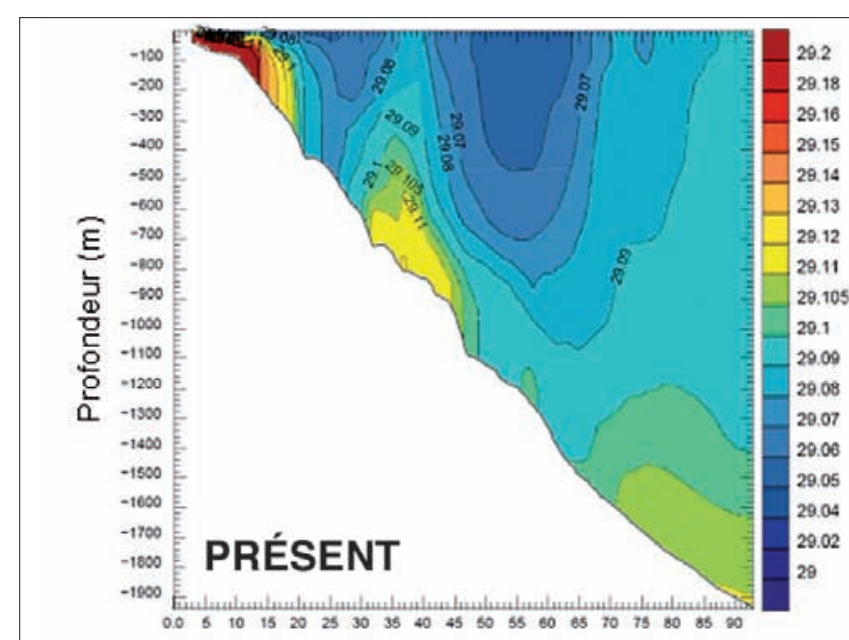

Distance à la côte $(\mathrm{km})$
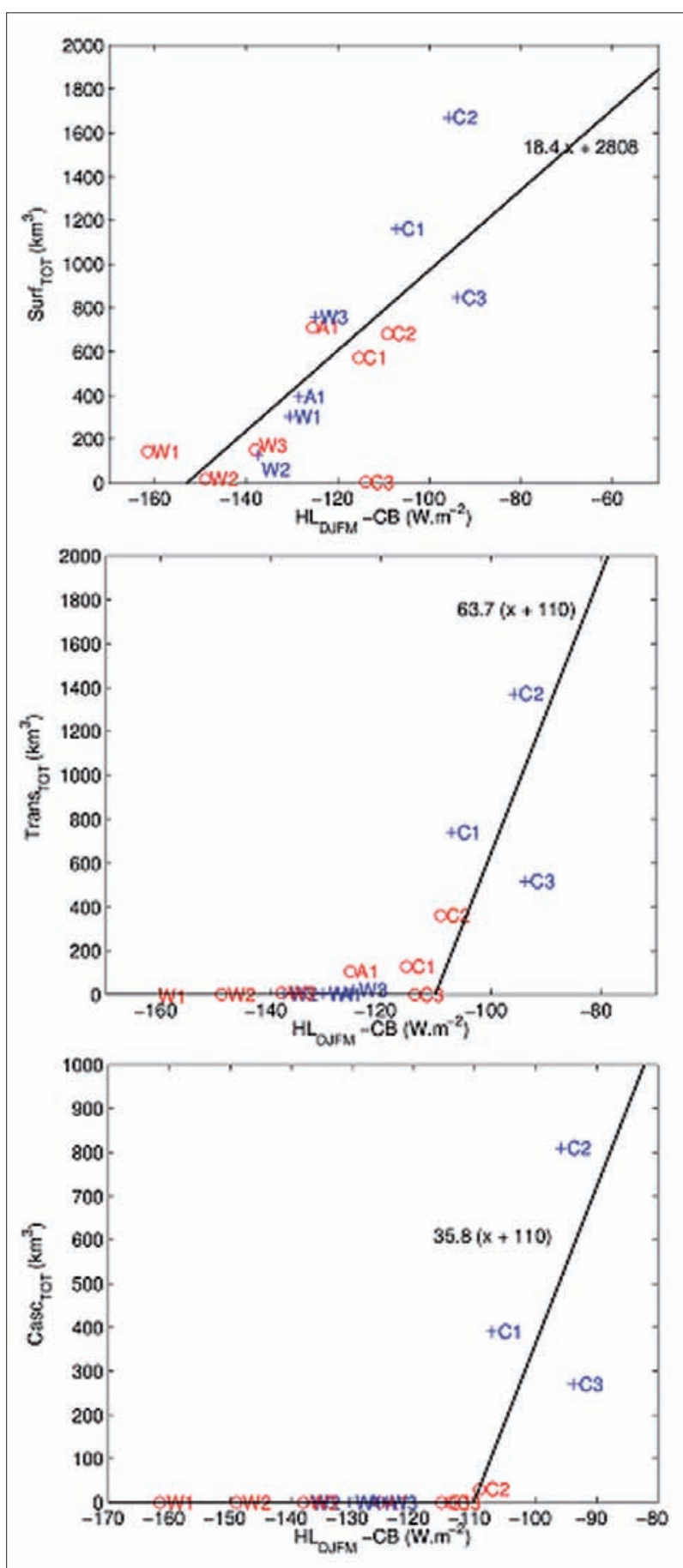

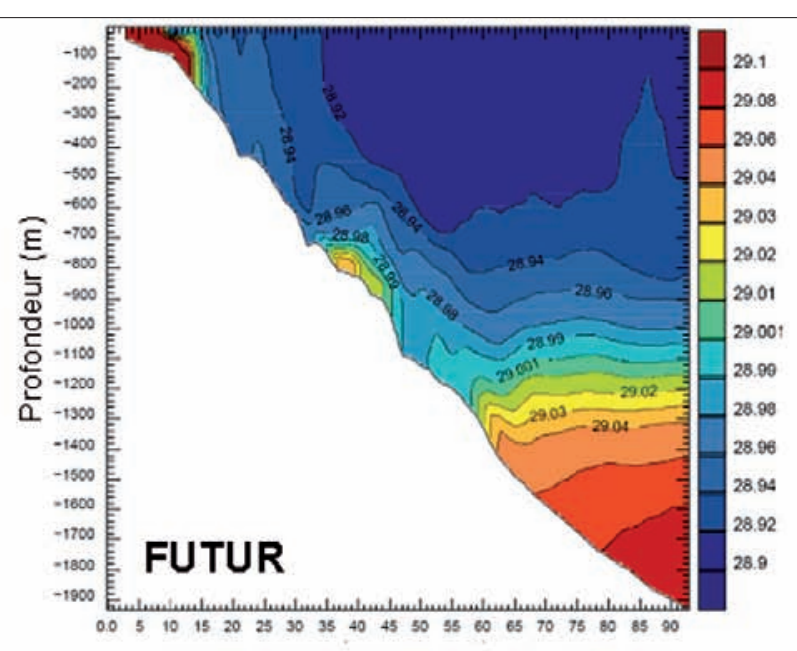

Distance à la côte (km)

hydrologique sont ceux d'EPA2. Pour des raisons de coût informatique - il n'était pas réaliste de réaliser une simulation de 140 ans - nous avons donc mené deux groupes de sept simulations : I'un pour la période présente (1970-2000) et l'autre dans la période future (2070-2100). L es années ont été sélectionnées à partir de la perte de chaleur atmosphérique hivernale dans le golfe du Lion : trois années plutôt chaudes, trois plutôt froides et une moyenne.

Sous le climat actuel, on observe dans les simulations une importante variabilité de la formation et du transport d'eau dense sur le plateau du golfe du Lion, avec des variations de deux ordres de grandeur. Les années de faible perte de chaleur, on n'a pratiquement pas

Figure 5 - Corrélation entre l'indice de flottabilité disponible et la formation, le transport plateau - large et le cascading (transport sous $200 \mathrm{~m}$ de profondeur) d'eau dense. Les années de la période actuelle sont indiquées en bleu et celles de période future en rouge.
Figure 4 - Coupe verticale de densité le long du canyon de Cap Creus (voir fig. 1) pendant un épisode de cascading lors d'une année de la période présente et une année de la période future. On observe une forte augmentation du gradient vertical de densité, donc de la stratification, entre les deux périodes.

d'eau dense formée, et le peu d'eau dense est exporté en surface (au-dessus de 200 mètres de profondeur) le long de la côte catalane. Les années de forte perte de chaleur, plus de la moitié de l'eau dense « cascade » vers le fond, le long du canyon du Cap Creus, sous 200 mètres de profondeur.

Sous le climat futur, on observe une nette diminution de la formation et de l'export d'eau dense. On n'observe pratiquement plus de cascading, la plus grande partie du peu d'eau dense formée est exportée en surface. Cette diminution ne peut être attribuée à un adoucissement des conditions hivernales : au contraire, la perte de flottabilité hivernale augmente entre la fin $d u x x^{e}$ et la fin $d u x x I^{e}$ siècle de $0,7 \times 10^{-8} \mathrm{~m}^{2} . \mathrm{s}^{-3}$, correspondant à une augmentation de la perte de chaleur de $30 \mathrm{~W} \cdot \mathrm{m}^{-2}$. En fait, la diminution de formation d'eau dense est liée à l'intensification de la stratification au cours du $x x I^{e}$ siècle, elle-même due à la diminution de la perte de flottabilité atmosphérique annuelle (Somot et al., 2006). L'influence de cette intensification de la stratification peut être estimée en calculant la perte de chaleur nécessaire pour densifier l'eau sur le plateau jusqu'à la densité de l'eau présente au large au fond : celle-ci augmente de $1,4 \times 10^{-8} \mathrm{~m}^{2} . \mathrm{s}^{-3}$, soit deux fois I'augmentation de la perte de flottabilité atmosphérique hivernale. Par conséquent, il est plus « difficile » pour l'eau dense formée à la surface $d$ ' atteindre une densité suffisante pour gagner le fond, comme on peut l'observer sur la figure 4. 
Conditions atmosphériques et stratification sont donc deux facteurs majeurs à l'origine de la variabilité et de l'évolution de la formation d' eau dense. Ces résultats nous ont conduits à construire un indice de perte de flottabilité qui tienne compte de ces deux facteurs: la différence entre la perte de flottabilité hivernale et la perte de flottabilité nécessaire pour amener la densité de l'eau de surface jusqu'à la densité de fond. Cette différence correspond en fait à la perte de flottabilité réellement disponible pour produire de l'eau dense à la surface. U ne relation linéaire est obtenue entre les quantités d'eau dense formée à la surface et exportée en profondeur, d'une part, et cet indice de perte de flottabilité disponible, d'autre part (figure 5 ). Ces relations sont utilisées pour extrapoler les résultats obtenus pour les deux groupes de sept années à toute la période présente (1970-2000) et future (20702100). Les résultats sont présentés sur

\section{0-2000 2070-2100}

\begin{tabular}{|c|c|c|}
\hline $\begin{array}{l}\text { Formée } \\
\text { à la surface }\end{array}$ & 7,9 & 4,1 \\
\hline Exportée & 6,0 & 0,6 \\
\hline $\begin{array}{l}\text { Exportée } \\
\text { sous } 200 \text { m } \\
\text { (cascading) }\end{array}$ & 3,5 & 0,3 \\
\hline
\end{tabular}

Tableau 1 - Quantités annuelles d'eau dense formée sur le plateau du golfe du Lion, exportée vers le large, et exportée sous 200 mètres de profondeur pour les périodes 1970-2000 et 2070-2100. Unité : $10^{11} \mathrm{~m}^{3}$. le tableau 1. A u final, la quantité d'eau dense formée à la surface diminue de $50 \%$ au cours du $\times \times I^{\mathrm{e}}$ siècle, et le cascading diminue de $90 \%$.

\section{Influence de la dynamique océanique sur les écosystèmes}

L'étude des simulations effectuées avec modèle Symphonie a montré que ce modèle reproduit correctement la dynamique océanique en $\mathrm{MNO}$, de la mésoéchelle au courant de bord (le courant nord). II est donc légitime de I'utiliser afin d'étudier l'influence de la dynamique sur le fonctionnement et l'évolution de l'écosystème pélagique planctonique dans cette région.

\section{Le modèle biogéochimique Eco3M-MED}

Une description détaillée d'Eco3M-MED est fournie dans Herrmann (2007), nous en rappelons ici les principales caractéristiques. II s'agit d'un modèle de type multinutriments et multigroupes fonctionnels décrivant la dynamique du plancton pélagique. II contient trente-trois variables d'état et prend en considération les cycles biogéochimiques du carbone, de l'azote, du phosphore et du silicium. La chlorophylle issue du phytoplancton est aussi représentée. Les groupes fonctionnels représentés sont : le zooplancton, divisé en trois groupes (mésozooplancton, microzooplancton et nanozooplancton) ; le phytoplancton (microphytoplancton, nanophytoplancton et picophytoplancton) ; les bactéries hétérotrophes ; la matière organique particulaire MOP ; la matière organique dissoute MOD ; les sels nutritifs ou matière inorganique dissoute MID (nitrate, ammonium, phosphate, silicate). Un schéma de synthèse du modèle est proposé figure 6 ci-dessous.

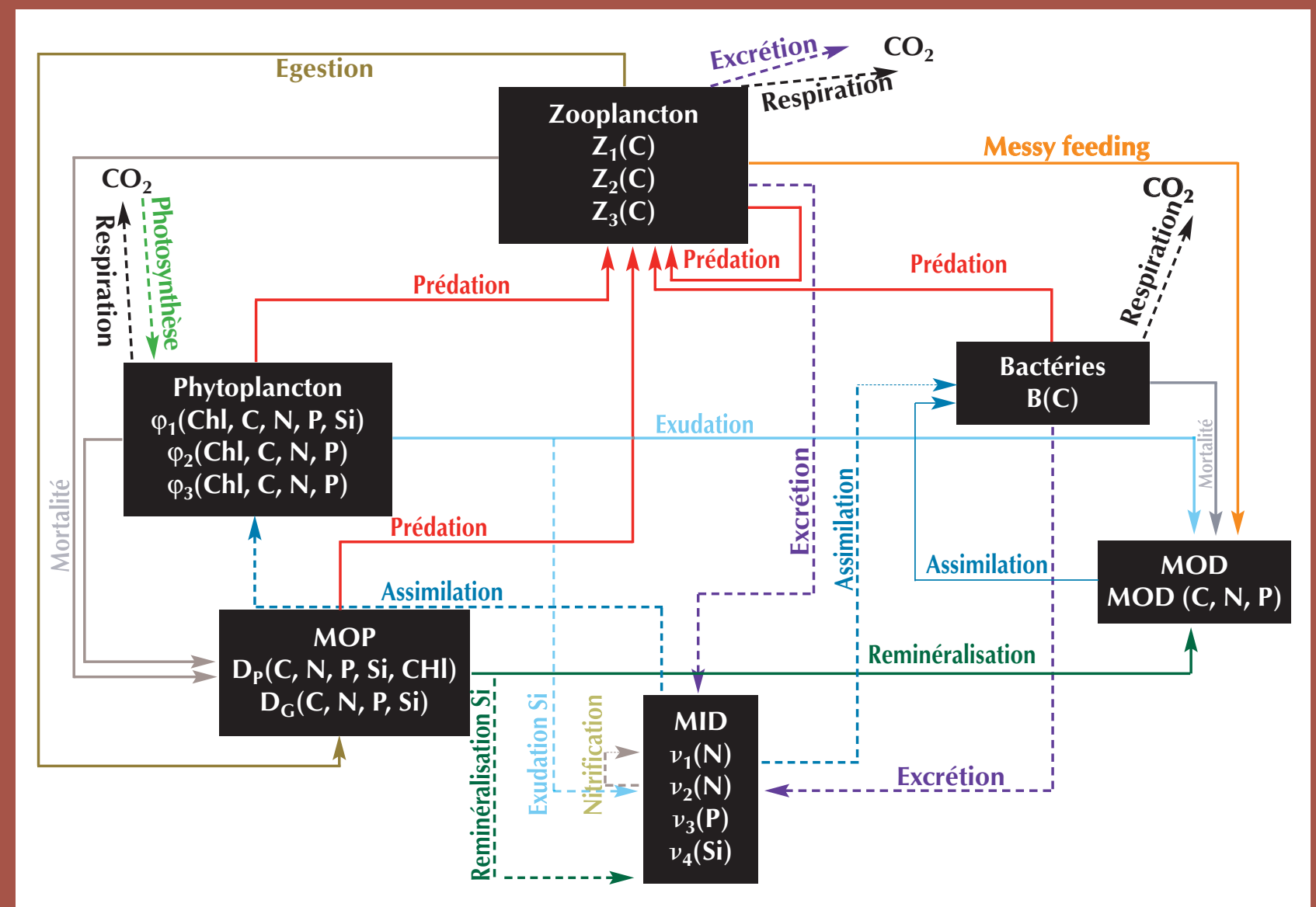




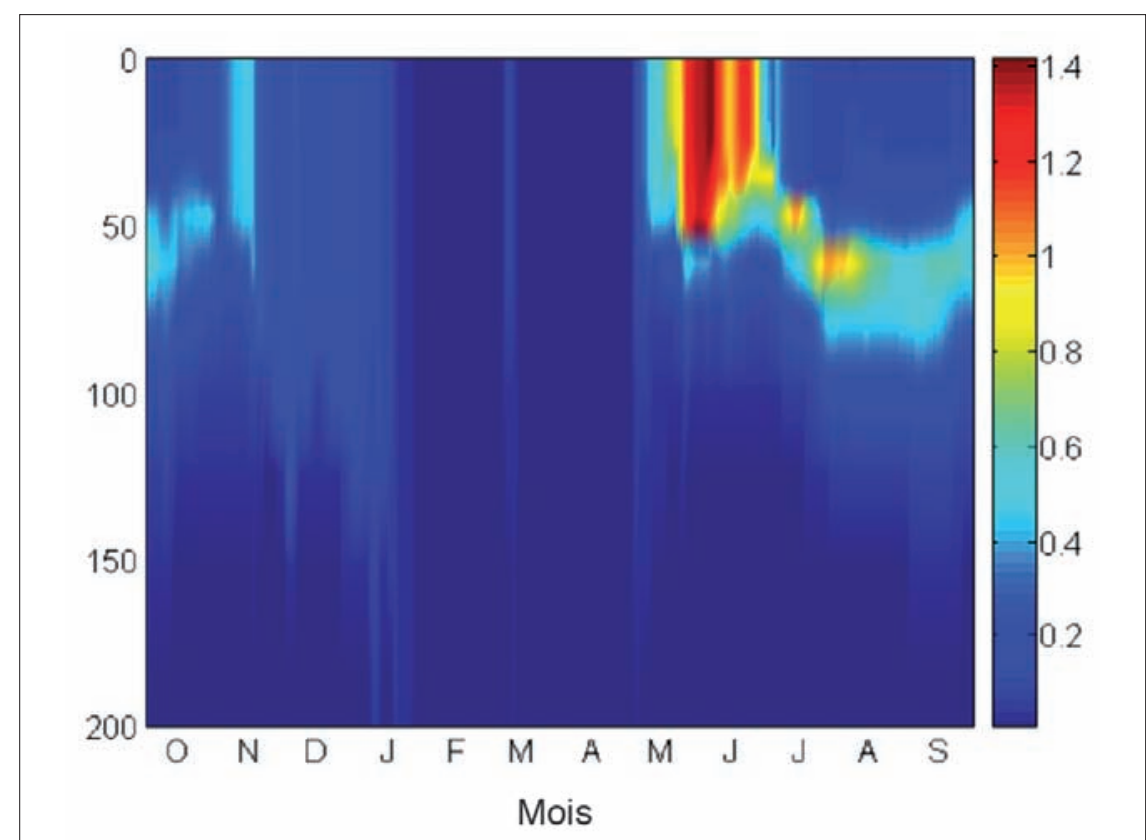

Figure 7 - Évolution de la concentration en chlorophylle pendant un an au centre de la région de convection (point $42^{\circ} \mathrm{N}, 5^{\circ} \mathrm{E}$ ) entre 0 et 200 mètres de profondeur. Unité : mgChl. $\mathrm{m}^{-3}$

\section{Le modèle couplé dynamique - biogéochimie régional}

Un couplage a été effectué entre le modèle de circulation régionale Symphonie et le modèle biogéochimique Eco3M-MED (voir encadré). E CO3M-MED décrit la dynamique du plancton pélagique et prend en considération les cycles biogéochimiques du carbone, de l'azote, du phosphore et du silicium. On utilise la même stratégie de modélisation que pour l'étude du cascading : les quatorze années des périodes actuelles et futures déjà étudiées du point de vue dynamique donnent lieu à quatorze simulations couplées physique-biogéochimie.

\section{Fonctionnement \\ de l'écosystème et cycle du carbone sur une année}

L'étude d'une simulation annuelle de la période actuelle nous permet d'abord de valider le modèle et d'identifier ses points faibles, puis d'étudier le fonctionnement de l'écosystème pélagique planctonique en M NO et son rôle dans le cycle du carbone.

Le modèle couplé reproduit correctement les grandes caractéristiques de I'écosystème. Premièrement, le modèle reproduit correctement I'évolution spatio-temporelle de l'écosystème (figure 7) : minimum de Production primaire brute PPB (correspondant à la fixation de gaz carbonique dissous pendant la photosynthèse) durant la convection hivernale ; maximum lors de l' efflorescence printanière, associé à de la production nouvelle (reposant sur I'apport exogène de nutriments) en surface ; PPB plus faible pendant I'été et l'automne, reposant sur la production régénérée (utilisation de nutriments régénérés dans la zone euphotique, c'est-à-dire où a lieu la photosynthèse, par les bactéries) en subsurface. La boucle microbienne est très active pendant I'été, et les bactéries sont responsables des trois quarts de la respiration (associée au rejet de gaz carbonique dissous) annuelle totale. Par ailleurs, le modèle couplé est capable de reproduire l'une des singularités biogéochimiques de la M éditerranée : le contrôle du développement phytoplanctonique et de la croissance bactérienne par la disponibilité en phosphore, pendant la période stratifiée.

Le nanophytoplancton est sensiblement surestimé par rapport aux autres groupes, en particulier en période estivale vis-à-vis du picophytoplancton, ce qui pourrait mener à une surestimation de la PPB à cette période. On note également une sous-estimation de la représentation du microphytoplancton au moment de l'efflorescence phytoplanctonique.

Un schéma de synthèse du rôle de I'écosystème pélagique planctonique dans le cycle du carbone en MNO est présenté figure 8. L e métabolisme net, qui correspond à la différence entre fixation de carbone par la PPB et rejet de carbone par la respiration, montre qu'à I'échelle annuelle I'écosystème planctonique en MNO constitue un « puits » pour le gaz carbonique dissous. L'exportation de carbone organique vers l'océan profond représente $13 \%$ de la PPB et est due majoritairement à l'exportation sous forme dissoute (COD) lors de la convection hivernale. U ne grande partie du carbone organique exporté vers le fond est ensuite exportée hors de la zone. Ce carbone est alors séquestré en profondeur pour au moins une centaine d'années (le temps de séjour des masses d'eau profonde en M éditerranée, Lacombe et al., 1981). La M NO étant la zone la plus productive du bassin ouest, la quantité totale de carbone organique séquestrée, égale au total à 6,6 M tC.an-1, donne donc un ordre de grandeur de la capacité de séquestration du carbone par l'écosystème planctonique du bassin ouest-méditerranéen, dont la surface représente $2 \%$ de l'océan global : la pompe biologique du bassin occidental méditerranéen serait responsable de $4 \%$ de la séquestration du carbone atmosphérique par l'océan global, qui s'élève à 1,8 G tC .an-1, pompes physique et biologiques incluses (Denman et al., 2007).

\section{Influence de la variabilité interannuelle atmosphérique et océanique}

La comparaison des sept années de la période actuelle, résumée sur la figure 8, permet d'examiner la variabilité de l'écosystème pélagique planctonique en M NO.

L'intensité de la convection hivernale, donc du mélange vertical, a une influence majeure sur la disponibilité en nutriments le reste de l'année : la taille de la zone enrichie en nutriments présente une variabilité de $34 \%$, positivement corrélée (coefficient de corrélation : 0,92) avec la profondeur moyenne de la couche de mélange au moment du maximum de convection. Cependant, les bilans annuels présentent globalement une variabilité interannuelle assez faible comparée à la variabilité de la disponibilité en nutriments. Les bilans annuels de PPB et de respiration sont corrélés positivement à la température moyenne de la zone euphotique (coefficient de corrélation : $0,95)$, et présentent une variabilité interannuelle très faible (respectivement $3 \%$ et $4 \%$ ). Le métabolisme net 


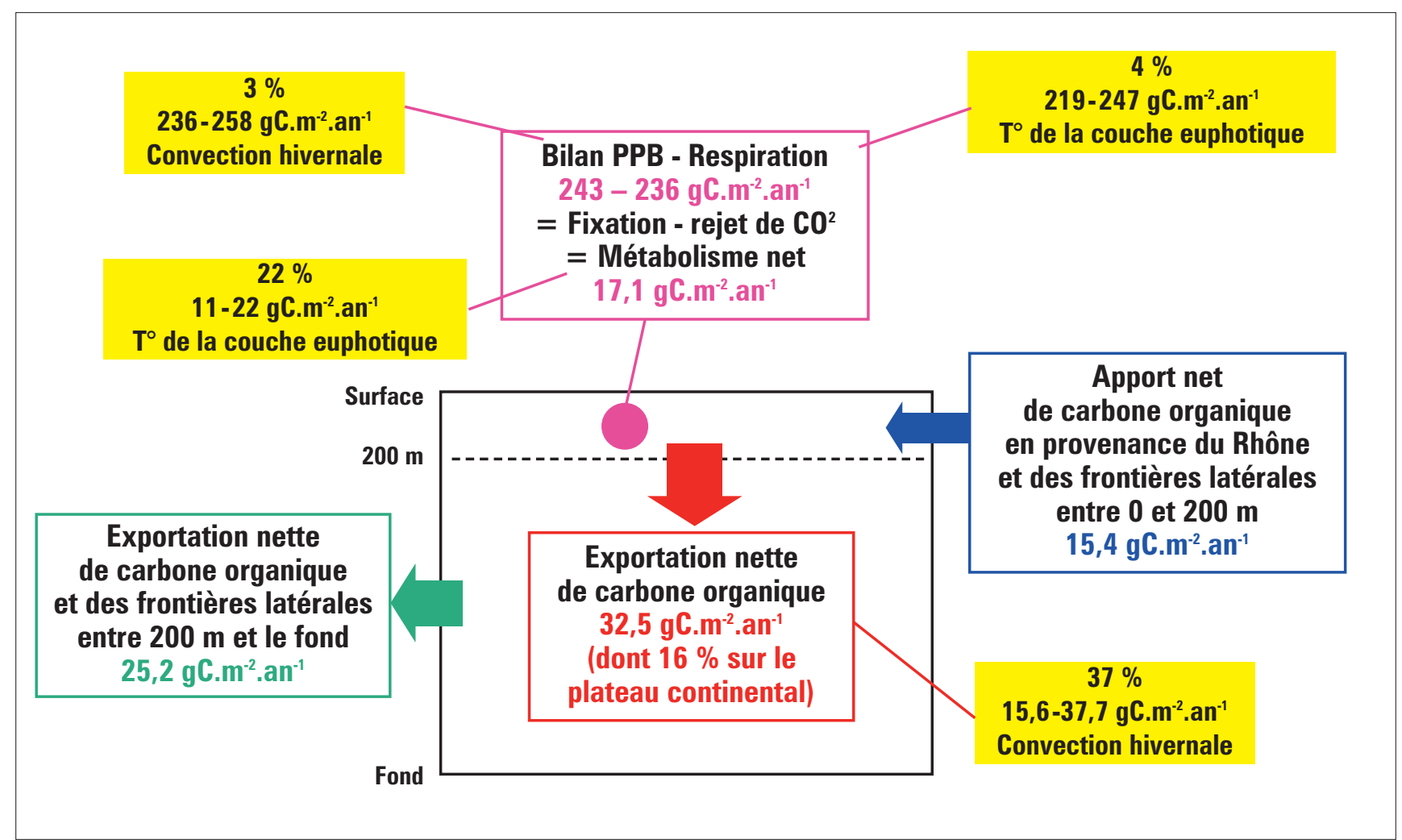

Figure 8 - Le cycle du carbone dans l'écosystème pélagique planctonique de MNO. Les valeurs dans les cadres de couleur correspondent au cycle annuel moyen. Les encadrés jaunes indiquent la variabilité interannuelle en pourcentage et en gamme de valeurs et le processus physique auquel est corrélée cette variabilité.

est corrélé négativement à la température moyenne $(-0,92)$, et montre une variabilité plus importante ( $22 \%)$. La variabilité de l'exportation de carbone organique vers le fond, liée à l'intensité et la durée de la convection hivernale, est également plus importante (37\%). Enfin, la part de la production régénérée par rapport à la production nouvelle, la biomasse bactérienne et la biomasse du picophytoplancton sont légèrement plus élevées les années chaudes, lorsque la convection et donc la disponibilité en nutriments provenant des couches profondes de l'océan sont plus faibles. Cela indique une plus forte activité de la boucle microbienne pendant ces années. L a limitation du dével oppement phytoplanctonique et de la croissance bactérienne par la disponibilité en phosphore, en particulier pendant la période stratifiée, est observée pour chaque année et est donc une spécificité marquée de l'écosystème planctonique pélagique en M NO.

De façon générale, on observe donc une faible variabilité interannuelle du fonctionnement de l'écosystème. Cela peut paraître surprenant ; on aurait par exemple pu s'attendre à observer un maximum de chlorophylle nettement plus marqué lors des années fortement convectives. II est cependant difficile de conclure plus définitivement sur ce point, en raison du peu d'observations disponibles. Cela souligne la nécessité de mettre en place des systèmes d'observation à long terme, mais aussi de valider et de calibrer plus précisément le modèle biogéochimique.

\section{Influence du changement climatique}

L a comparaison des résultats obtenus lors de chaque groupe des sept années des périodes actuelle et future permet d'évaluer I'influence du changement climatique sur l'évolution saisonnière et la composition de l'écosystème planctonique pélagique en $\mathrm{MNO}$ et son rôle dans le cycle du carbone, sous les hypothèses du scénario $A 2$. L es résultats obtenus sont synthétisés sur la figure 9 .

L a disponibilité en nutriments diminue entre la période actuelle et la fin du $x \times I^{\mathrm{e}}$ siècle [-32\%], en raison de I'affaiblissement de la convection océanique profonde [-52\%], (flèche 1 sur la figure 9). Paradoxalement, la PPB, qui dépend de la température, augmente [ $+19 \%$ ] en raison de la hausse de la température dans la zone euphotique $\left[+3,4{ }^{\circ} \mathrm{C}\right]$ (flèche 2 ). En conséquence de la plus faible disponibilité en nutriments et de I'augmentation de la production primaire, l'exudation phytoplanctonique de carbone organique dissous augmente [+35\%] (flèche 3 ).
Cette augmentation de la concentration en carbone organique dissous favorise la croissance bactérienne [+15\%] (flèche 4), qui repose sur l'absorption de matière organique dissoute. L'excrétion bactérienne d'ammonium augmente alors, entraînant une très légère augmentation de la part de la production primaire liée à la production régénérée $[+1 \%]$. La hausse du rejet de gaz carbonique [ $+20 \%$ ] résultant en grande partie de la hausse de la respiration bactérienne (flèche 5 ) compense pratiquement I' augmentation de la PPB [+19\%] : la quantité nette de gaz carbonique fixée au cours du cycle annuel par le système augmente légèrement [+8 \%] (flèche 6). Enfin, l'exportation de carbone organique vers les couches profondes de la M NO diminue peu [-4 \%], I'affaiblissement de la convection océanique étant compensé par I' augmentation des quantités de carbone organique dissous et particulaire (flèche 7).

Ces résultats constituent une première étape de l'étude de la réponse de l'écosystème planctonique pélagique au changement climatique, qui apparaît finalement assez faible. Un travail important d' analyse et de réduction des différentes sources $d$ 'incertitudes reste cependant à faire. Rappelons d'abord que l'évolution des concentrations en nutriments sur les cent prochaines 


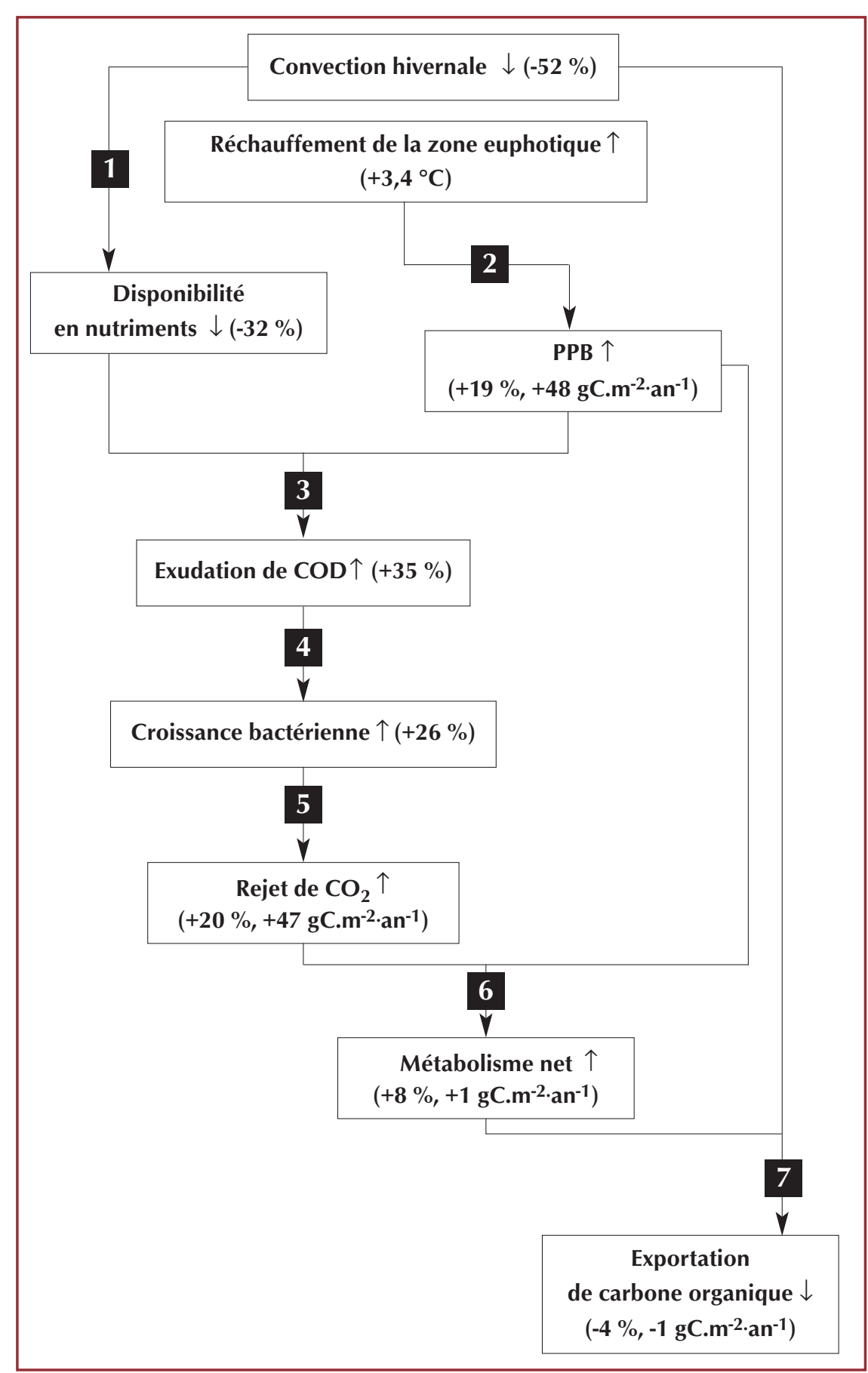

Figure 9 - Influence du changement climatique sur l'écosystème planctonique et le cycle du carbone en MNO. Les pourcentages indiquent la variation relative entre les périodes présente et future, et les valeurs en $\mathrm{gC} . \mathrm{m}^{-2} . \mathrm{an}^{-1}$ la variation absolue.

années, liée à l'évolution des apports fluviaux et atmosphériques, n'a pas été prise en compte. De plus, ce travail s'appuie sur une simulation de grande échelle, réalisée avec un modèle océanique et un modèle atmosphérique particuliers, sous les hypothèses d'un scénario de changement climatique particulier. Enfin, les formulations et les paramétrisations des processus ne permettent pas forcément au modèle biogéochimique de représenter tous les changements qui pourraient intervenir dans l'écosystème en réponse à l'évolution du climat : adaptation des espèces à
I'augmentation de la température, apparition de nouvelles espèces, changement de régime trophique, etc.

\section{Bilan et perspectives}

L a modélisation est un outil particulièrement adapté à l'étude de la formation et du devenir des masses d'eau dense : elle permet d'étudier les processus physiques qui entrent en jeu dans la convection profonde et le cascading, d'examiner leur réponse aux différents facteurs de variabilité d'origine naturelle (atmosphérique, océanique ou hydrologique) ou anthropique, et finalement d'étudier leur rôle dans le fonctionnement des écosystèmes marins et le cycle du carbone.

L'étude d'un cas réel de convection profonde a permis de quantifier le rôle fondamental des structures de mésoéchelle dans la formation et le devenir de la W M DW, et celui des événements atmosphériques intenses dans l'approfondissement de la couche de mélange. Ces résultats soulignent la nécessité d'utiliser des modèles océaniques et atmosphérique à haute résolution (respectivement au moins $3 \mathrm{~km}$ et $50 \mathrm{~km}$ ) pour représenter de façon réal iste les processus de formation et de propagation d'eau dense, que ce soit pour l'étude de cas particuliers ou pour examiner ces processus sur de longues périodes. Dans la continuité de ce travail, le projet M edup vise actuellement à estimer l'influence de la conf iguration des modèles atmosphériques sur la représentation des événements atmosphériques intenses (Herrmann et Somot, en préparation).

L'outil de modélisation mis en place a été ensuite utilisé pour étudier la réponse du cascading d'eau dense sur le talus continental du golfe du Lion aux variations océaniques et atmosphériques à plus ou moins long terme. Sous le climat actuel, la forte variabilité interannuelle de ce processus est largement corrélée à la perte de flottabilité hivernale. Le changement climatique induit une quasi-disparition du cascading d'ici à la fin du $\times \times I^{\mathrm{e}}$ siècle. Cette diminution n'est pas liée à un affaiblissement des conditions atmosphériques hivernales mais à une intensification de la stratification océanique due à la diminution de la perte de flottabilité atmosphérique annuelle au cours du $x \times I^{e}$ siècle. La prochaine étape sera de déterminer quelle pourrait être l'influence de ces variations de transport de masses d'eau sur les échanges côte-large de matière organique et particulaire et sur le cycle du carbone.

Enfin, nous avons réalisé ici une première étude de modélisation couplée physique-biogéochimie tridimensionnelle à mésoéchelle. Cela nous a permis d'examiner le rôle de l'écosystème pélagique planctonique de M NO dans le cycle du carbone. Sous le climat actuel, les résultats suggèrent que cet écosystème est un puits pour le gaz carbonique dissous. N otons cependant qu'il serait nécessaire d'inclure la 
pompe chimique dans le modèle pour conclure quant au rôle de la M NO visà-vis du carbone atmosphérique. La variabilité interannuelle de l'écosystème est relativement faible, avec tout de même une variabilité plus importante de l'exportation de carbone organique en profondeur, corrélée à l'intensité de la convection océanique hivernale. Enfin, les résultats suggèrent une réponse relativement faible au changement climatique, qui entraînerait une légère diminution de l'exportation de carbone ainsi qu'une intensification de la boucle microbienne. Cette étude montre que cette démarche de modélisation couplée est prometteuse, mais qu' un travail important reste à faire en ce qui concerne la validation, la calibration et I' amélioration du modèle ainsi que l'évaluation des différents facteurs d'incertitudes.

\section{Remerciements}

Je remercie Claude Estournel, du L aboratoire d'aérologie, de m'avoir guidée pendant ce travail de thèse, ainsi que Frédéric Diaz du Laboratoire d'océanographie physique et biogéo- chimique ( $L O P B$ ) pour son encadrement sur la partie dédiée à la biogéochimie. Merci également à toute l'équipe du Pôle d'océanographie côtière de Toulouse, en particulier à Caroline UIses pour son travail sur le modèle biogéochimique et à Patrick $M$ arsaleix pour son soutien et son expertise sur Symphonie. La collaboration avec Samuel Somot, Florence Sevault et $M$ ichel Déqué du Centre national de recherches météorologiques (CNRM) a également apporté énormément à ce travail. M erci enfin au jury du prix Prud'homme de m'avoir accordé ce prix.

\section{Bibliographie}

: Artale V., D. ludicone, R. Santoleri, V. Rupolo, S. Marullo et F. D’Ortenzio, 2002 : Role of surface fluxes in ocean general circulation models using satellite sea - surface temperature : Validation of and sensitivity to the forcing frequency of the Mediterranean thermohaline circulation. J. Geophys. Res., 107 (C8), DOl: 10.1029/2000JC000452.

- Béthoux J., X. Durrieu de Madron, F. Nyffeler et D. Tailliez, 2002 : Deep water in the Western Mediterranean: peculiar 1999 and 2000 characteristics, shelf forma-

tion hypothesis, variability since 1970 and geochemical inferences. J. Mar. Syst., 33-34, 117-131.

Canals M., P. Puig, X. Durrieu de Madron, S. Heussner, A. Palanques et J. Fabrès, 2006 : Flushing submarine canyons. Nature, 444, $354-357$.

- Denman K. L., G. Brasseur, A. Chidthaisong, P. Ciais, P. M. Cox, R. E. Dickinson, D. Hauglustaine, C. Heinze, E. Holland, D. Jacob, U. Lohmann, S. Ramachandran, P. L. da Silva Dias, S. C. Wofsy et X. Zhang, 2007 : Couplings between changes in the climate system and biogeochemistry. In: Climate Change 2007: The physical science basis. Contribution of Working Group I to the Fourth Assessment Report of the Intergovernmental Panel on Climate Change [Solomon S., D. Oin, - M. Manning, Z. Chen, M. Marquis, K. B. Averyt, M. Tignor et H. L. Miller (eds.)]. Cambridge University Press, Cambridge, Royaume-Uni and New York, NY, États-Unis.

- Herrmann M., 2007 : Formation and fate of water masses in the Northwestern Mediterranean Sea. Impact on pelagic planktonic ecosystems. Interannual variability and - climate change. Thèse de doctorat, université Toulouse III, 310 p.

Herrmann M., S. Somot, F. Sevault, C. Estournel et M. Déqué, 2008a : Modeling deep convection in the Northwestern Mediterranean Sea using an eddy-permitting - and an eddy-resolving model: case study of winter 1986-1987. J. Geophys. Res. 113, C04011, D0I: 10.1029/2006JC003991.

Herrmann M., C. Estournel, S. Somot, M. Déqué, P. Marsaleix et F. Sevault, 2008b : Impact of interannual variability and climate change on dense water cascading in the Gulf of Lions. Continental Shelf Research, 28 (15), 2092-2112, D0I: 10.1016/j.csr.2008.03.003.

- Herrmann M. et S. Somot, 2008 : Relevance of ERA40 dynamical downscaling for modeling deep convection in the North-Western Mediterranean Sea. Geophys. Res. Lett., 35, L04607, DOI: 10.1029/2007GL032442.

- Heussner S., X. Durrieu de Madron, A. Calafat, M. Canals, J. Carbonne, N. Desault et G. Saragoni, 2006 : Spatial and temporal variability of downward particle - fluxes on a continental slope: Lessons from an 8-yr experiment in the Gulf of Lions (NW Mediterranean). Marine Geology, 234, 63-92.

Ivanov V. V., G. I. Shapiro, J. M. Huthnance, D. L. Aleynik et P. N. Golovin, 2004 : Cascades of dense water around the world ocean. Prog. Oceanogr., $60,47-98$.

Kalnay E. et al., 1996 : The NCEP/NCAR 40-year reanalysis project. Bull. Amer. Meteor. Soc. , 77, 437-471.

- Lacombe H., J. C. Gascard, J. Gonella et J. P. Béthoux, 1981 : Response of the Mediterranean to the water and energy fluxes across its surface, on seasonal and interannual scales. Oceanologica Act, 4(2), 247-255.

- Leaman K. D. et F. Schott, 1991 : Hydrographic structure of the convection regime in the Gulf of Lions : Winter 1987. J. Phys. Oceanogr., 21, 575-597.

- Madec G., M. Chartier, P. Delecluse et M. Crépon, 1991 : A three-dimensional numerical study of deep-water formation in the Northwestern Mediterranean Sea. J. Phys. Oceanogr., 21(9), 1349-1371.

- Marshall J. et F. Schott, 1999 : Open-ocean convection: observations, theory, and models. Rev. Geophys., 37 (1), 1-64, D0I: 10.1029/98RG02739.

Medoc-Group, 1970 : Observations of formation of deep-water in the Mediterranean Sea. Nature, 227, 1037-1040.

- Mertens C. et F. Schott, 1998 : Interannual variability of deep-water formation in the Northwestern Mediterranean. J. Phys. Oceanogr., 28, 1410-1424.

- Schott F. et K. D. Leaman, 1991 : Observations with moored acoustic doppler current profilers in the convection regime in the Golfe du Lion. J. Phys. Oceanogr., 21, 558-574.

- Send U., J. Font et C. Mertens, 1996 : Recent observations indicates convection's role in deep circulation. E0S T. Am. Geophy. Un., 77, 61-65.

- Somot S., F. Sevault et M. Déqué, 2006 : Transient climate change scenario simulation of the Mediterranean Sea for the 21 st century using a high resolution ocean circulation model. Clim. Dyn., 27 (7-8), 851-879, D0I: 10.1007/s00382-006-0167-z.

Testor P. et J.-C. Gascard, 2006 : Post-convection spreading phase in the Northwestern Mediterranean Sea. Deep-Sea Res., Part I, 53, 869-893. 